\title{
Evaluation des besoins en prothèse dentaire chez les patients au service d'hépato-gastroentérologie du CHU-Gabriel Touré de Bamako
}

\section{Assessment of dental prosthesis needs of patients in the service of hepato-gastroenterology CHU- Gabriel Toure of Bamako}

\author{
Coulibaly $\mathrm{B}^{1 *}$, Kéita DB${ }^{1}$, Touré $\mathrm{K}^{1}$, Kamissoko $\mathrm{K}^{2}$, Diallo $\mathrm{B}^{1}$, Traoré $\mathrm{L}^{2}$, Kané AST${ }^{2}$, Traoré $\mathrm{H}^{1}$, Diarra MT³
}

${ }^{1}$ Centre Hospitalier Universitaire Centre National d'Odontostomatologie (CHU-CNOS)

2Direction Centrale des Services de Santé des Armées (DCSSA)

${ }^{3}$ Centre Hospitalier Universitaire Gabriel Touré (CHU-Gabriel Touré)

*Correspondant : Dr Coulibaly Bougadadry, Chirurgien-dentiste Prothésiste, Praticien Hospitalier au CHU CNOS, Maitre-Assistant à la FMOS.

\section{RESUME}

Introduction : La santé bucco-dentaire est caractérisée par l'absence de douleur buccale ou faciale, de cancer de la bouche ou du pharynx, d'infection ou de lésion buccale, de maladie parodontale, de déchaussement et de perte de dents et d'autres maladies et troubles qui limitent la capacité de mordre, mâcher, sourire et parler d'une personne, et donc son bien-être psychosocial. L'édentement étant un manque partiel ou total de dents, il réduit à lui seul la capacité de mordre, de mâcher, de sourire, de parler et de bien-être psychosocial. L'objectif général de cette étude était d'identifier les besoins de réhabilitation prothétique dentaire chez les patients hospitalisés âgés de 15 ans et plus dans le service d'hépato-gastro-entérologie du CHU Gabriel Touré de Bamako. Matériel et Méthodes : II s'agissait d'une étude transversale descriptive qui s'est déroulée sur une période de 3 mois du 18 août au 18 novembre 2016 dans le service d'hospitalisation de l'unité d'hépato-gastro-entérologie du CHU Gabriel Touré de Bamako. Ont été inclus, tous les patients hospitalisés au service d'hépato-gastroentérologie du CHU-Gabriel Touré, âgés de 15 ans ou plus ayant répondu aux questionnaires. Nous avons colligé 100 patients. Résultats : Dans notre étude, nous avons une prédominance masculine avec $58 \%$. La tranche d'âge des 45 à 54 ans représentait $25 \%$ de la population active avec une moyenne de 44 ans. La présence de tartre est observée chez $70 \%$ de nos patients; la candidose était plus représentée avec $57 \%$ des cas. Sur le plan hépatogastro-entérologique, les maladies hépatiques étaient les plus représentés avec $37 \%$. L'étude a rapporté $64 \%$ des cas d'édentement avec au moins une dent manquante. La région molaire était la plus représentée dans $82,81 \%$ des cas. Conclusion : Les édentements constituent un problème de santé publique en raison de leur prévalence et de leur handicap. La réhabilitation prothétique dentaire rétablit l'équilibre biologique, fonctionnel, esthétique et psychologique.

Mots clés : bilan, besoins, prothèse dentaire, hépatogastro-entérologie, CHU-Gabriel Touré, Bamako

\section{ABSTRACT}

Introduction: Oral health is characterized by the absence of oral or facial pain, cancer of the mouth or pharynx, infection or lesion of the mouth, periodontal disease, loosening and loss of teeth and other diseases and disorders that limit a person's ability to bite, chew, smile and speak, and therefore their psychosocial well-being. Edentulousness being a partial or total lack of teeth, it alone reduces the ability to bite, chew, smile, speak and psychosocial well-being. The general objective of this study was to identify the needs for dental prosthetic rehabilitation in hospitalized patients aged 15 years and over in the hepato-gastroenterology department of the CHU Gabriel Touré in Bamako. Materiel and Methods: This was a descriptive cross-sectional study which took place over a period of 3 months from August 18 to November 18, 2016 in the inpatient department of the hepato-gastroenterology unit of the $\mathrm{CHU}$ Gabriel Touré in Bamako. All patients hospitalized in the hepatogastroenterology department of the CHU-Gabriel Toure, aged 15 years or over, who answered the questionnaires were included. We have collected 100 patients. Results: In our study, we have a male predominance with $58 \%$. The 45 to 54 age group represented $25 \%$ of the working population with an average of 44 years. The presence of tartar is observed in $70 \%$ of our patients; candidiasis was more represented with $57 \%$ of cases. On the hepatogastroenterological level, hepatic diseases were the most represented with $37 \%$. The study reported $64 \%$ of cases of edentulousness with at least one missing tooth. The molar region was the most represented in $82.81 \%$ of cases. Conclusion: Edentulousness is a public health problem due to its prevalence and disability. Dental prosthetic rehabilitation restores the biological, functional, aesthetic and psychological balance.

Keywords: assessment, needs, dental prosthesis, hepato-gastroenterology, Teaching Hospital Gabriel Toure, Bamako

\section{INTRODUCTION}

Selon l'Organisation Mondiale de la Santé (OMS), la santé est un état complet de bien-être physique, mental et social, et ne consiste pas seulement en une absence de maladie ou d'infirmité [1].

La santé bucco-dentaire se caractérise par l'absence de douleur buccale ou faciale, de cancer buccal ou pharyngé, d'infection ou de lésion buccale, de parodontopathie, de déchaussement et perte de dents, et d'autres maladies et troubles qui limitent la capacité de mordre, mâcher, 
sourire et parler d'une personne, et donc son bien-être psychosocial [2].

L'édentement étant un manque dentaire partiel ou total, il réduit à lui seul la capacité de mordre, de mâcher, de sourire, de parler et le bien-être psychosocial. II cause ainsi d'autres conséquences sur l'architecture musculaire et osseuse de la face.

L'appareil masticateur devait être complet afin de demeurer en bonne santé et fournir une fonction adéquate et satisfaisante [2]. L'OMS prévoit 30\% d'édentés totaux dans le monde en 2030 et cette pathologie concerne environ $2,3 \%$ de la population mondiale en 2010 [3].

La majorité des arguments épidémiologiques implique une relation de cause à effet entre la mastication et la digestion sur les données obtenues chez les sujets édentés et porteurs de prothèses dentaires inefficaces. Une première étude réalisée à Porto-Rico en 1947 par Rodriguez-Olleros rapportait que $48 \%$ des 168 patients édentés souffraient de gastrite décelable radiologiquement, seul $6 \%$ des sujets ayant une mastication adéquate présentaient la gastrite [4]. St Nikolov et al. lors d'endoscopie réalisée chez 92 sujets édentés ont constaté des anomalies de l'histologie gastrique chez presque tous [5]. Berndt dans une revue analysant les facteurs associés à la gastrite chronique atrophique mentionne une association entre la durée du déficit masticatoire et la sévérité des anomalies gastriques [6].

L'intérêt de notre étude réside dans le fait que, la santé bucco-dentaire est étroitement liée à l'état de santé générale et au bien-être ; la cavité buccale est un indicateur de bonne santé ou de maladie et ce par la richesse des pathologies muqueuses locales, leur contribution aux pathologies générales et leur extension à travers les « annexes » de cette cavité orale ; elle est aussi le premier segment de l'appareil digestif et les dents sont les éléments les plus spécialisés du système masticateur. L'objectif général de cette étude était d'identifier les besoins en réhabilitation prothétique dentaire chez les malades hospitalisés âgés de15 ans et plus au service d'hépato-gastro-entérologie du Centre HospitaloUniversitaire Gabriel Touré de Bamako.

\section{MATERIEL ET METHODOES}

II s'agissait d'une étude descriptive de type transversale qui s'est déroulée sur une période de 3 mois, allant du 18 Aout au 18 Novembre 2016, au service d'hospitalisation de l'unité d'hépato-gastro-entérologie du CHU Gabriel Touré de Bamako.

Etaient inclus tous les malades volontaires et consentants, hospitalisés dans le service d'hépatogastro-entérologie du CHU-Gabriel Touré d'âge supérieur ou égal à15 ans, ayant répondu aux questionnaires.

Etait exclu, tout malade hospitalisé dans le service pour autres pathologies non hépato-gastroentérologiques, ayant pas 15 ans et ceux qui n'ont pas accepté de répondre aux questionnaires. II s'agissait d'un recrutement de façon exhaustive sur les patients hospitalisés au service d'hépato-gastro-entérologie du CHU- Gabriel Touré de Bamako au cours de laquelle nous avons inclus 100 patients. Les matériels suivants ont été utilisés : Plateaux ; Miroirs (plan buccal) ; Sondes (simples) ; Désinfectants (hypochlorite de sodium et gel alcoolisé) ; Gants stériles.

Les matériels sortaient de la stérilisation chaque matin, après examen de chaque patient. Ils étaient décontaminés par lavage à la brosse avec du savon puis désinfectés dans une cuvette contenant une préparation de solution d'hypochlorite de sodium faite selon la méthode de Virginia Anderson (une part d'eau de javel pour six parts d'eau de robinet).

Les patients ont été informés des buts de notre étude et de la confidentialité des informations obtenues. L'enquête a été effectuée à travers des questionnaires anonymes. Les examens cliniques individuels ont été réalisés après avoir obtenu le consentement verbal éclairé des patients. Les données ont été saisies sur EXCEL 2010 et analysées sur EPI INFO version 3.5.4.

\section{RESULTATS}

Le sexe masculin était le plus représenté avec $58 \%$ des cas. Le sex ratio était de 1,38 (H/F) (tableau I).

La tranche d'âge la plus représentée était de 45 à 54 ans avec $25 \%$. La moyenne d'âge était de 44 ans $\pm 15,32$. Les extrêmes de 19 à 90 ans et un écart type (tableau II).

Les pathologies hépatiques et gastriques ont été les plus représentées dans les différents groupes de dents affectés suivies des VIH et opportunistes (tableau III).

$>$ Port de prothèse :

Notre étude fait état de 11 malades porteurs de prothèse amovibles partielles et aucun porteur de prothèses conjointes ; soit $11 \%$ de notre échantillon, $36 \%$ avec une denture complète et $53 \%$ d'édentés non porteurs de prothèse.

Les patients atteints de pathologies gastriques ont été les plus représentés avec $54,5 \%$ des cas (tableau IV).

$>$ Besoin diagnostiqué

Notre étude nous montre que le besoin en prothèse est diagnostiqué chez $64 \%$ de l'échantillon.

\section{DISCUSSION}

Dans notre étude nous avons une prédominance masculine de 58\% avec un sex ratio de 1,38 (H/F). Ce résultat est supérieur à celui de $\mathrm{KA} K$ au Sénégal issu d'une enquête sur la population Dakaroise qui rapporte une prédominance masculine de $53,3 \%$ [7]. Cette différence pourrait s'expliquer par la taille de l'échantillon. La tranche d'âge de 45 à 54 ans a été la plus représentée avec $25 \%$ de l'effectif. La moyenne des âges était de 44 ans avec un écart type de 15,32\%. Cette représentation peut s'expliquer parce que cette tranche est beaucoup exposée aux facteurs de risque des affections hépato- 
gastro-entérologiques tels que les habitudes alcoolotabagiques et la prise d'anti-inflammatoires non stéroïdiens [8].

La présence de tartre est observée chez $70 \%$ de nos patients ; Camara $S$ dans son étude a évoqué la présence de tartre chez $49 \%$ des patients. Ceci peut avoir comme explication la mauvaise habitude d'hygiène buccodentaire.

Selon les types de lésions rencontrées, la candidose a été plus représentée avec $57 \%$ des cas. Ceci explique la répercussion des pathologies générales principalement des pathologies digestives sur la muqueuse buccale et une défaillance des mesures d'hygiène bucco-dentaire. Selon les pathologies hépato-gastro-entérologiques, les cas d'hépatopathies ont été les plus représentés avec $37 \%$. Ce résultat confirme une prévalence élevée et une forte fréquence d'hospitalisation liée aux pathologies hépatiques. Le Mali fait parties des pays les plus touchés par cette pandémie [9].

Nous avons noté une absence de prothèse conjointe et de prothèse amovible complète. Ces résultats sont en discordance avec ceux de KA K. qui rapporte une dominance de prothèse conjointe sur la prothèse amovible. Les femmes aux foyers étaient les plus représentées des porteurs dans $36,4 \%$ des cas. Ce résultat confirme l'attachement des femmes aux foyers à la prothèse à cause de l'esthétique.

Selon les pathologies, les $54,5 \%$ des porteurs étaient atteints de pathologies gastriques. Ces résultats montrent qu'il existe une relation entre le port de prothèse et la pathologie gastrique. L'étude de Faye $D$ et al a permis de voir une association significative entre l'état dentaire et la pathologie digestive [12].

Besoin en prothèse : Le besoin diagnostiqué et justiciable en prothèse est observé chez $64 \%$ des cas contre $11 \%$ qui en portaient effectivement. Mbodj E B et al dans leur étude ont diagnostiqué un besoin en prothèse dans $81,8 \%$ des cas [13]. En fonction du sexe, le besoin s'élève à $71,43 \%$ chez les femmes contre $58,62 \%$ chez les hommes.

\section{CONCLUSION}

L'édentement constitue un problème de santé publique par sa prévalence et son handicap. La réhabilitation prothétique dentaire permet de restaurer l'équilibre biologique, fonctionnel, esthétique et psychique et aussi de protéger les dents restantes sur l'arcade. L'état d'absence dentaire non restauré se traduit par une privation de la cavité buccale de ces éléments spécialisés et une perturbation du processus digestif par une mauvaise mastication.

Notre étude nous a permis de découvrir une déficience des mesures d'hygiène bucco-dentaire, un énorme besoin en soins conservateurs et en réhabilitation prothétique dentaire dans la population examinée. Ainsi devant ces résultats, il convient d'organiser des séances d'information, d'éducation et de communication, de fluorations, de dépistages et de traitements préventifs en vue de renforcer les politiques de santé bucco-dentaire existantes.

\section{REFERENCES}

1. OMS. Préambule à la constitution mondiale de la santé. New York, 19-22 juin 1946(Acte officiel de l'OMS $\left.n^{\circ} 2 p 100\right)$ 2- OMS. Santé buccodentaire (aide-mémoire $N^{\circ} 318$ Avril 2012).

2. OMS. Santé bucco-dentaire (aide-mémoire $\mathrm{N}^{\circ}$ 318 Avril 2012)

3. OMS. Rapport mondial sur la santé buccodentaire (24 février 2004/Genève).

4. Wikipédia. Edentement. https://fr.m.wikipédia.org ». (28 février 2017 à 14:47).

5. Rodriguez-Olleros A.Gastritis in the toothless. Gastoenterol1947;14: 180-6.

6. St Nikolov E,Seniov M, Kaleva M. Reflection of alveolar ridge defects on the gastrointestinal tract. Stomatol (Sofia) 1970; $52: 389-94$.

7. Berndt $\mathrm{H}$. Epidemiologycal approaches to the aetiology of chronic atrophic gastritis. Digestion $1971 ; 4: 250-4$

8. KaK. Evaluation des besoins en prothèse dentaire dans la commune de Dakar. Thèse chir dent, Dakar, 2002 ; 17. 63p.

9. Napon, Zongo P, Delphine. Anti-inflammatoire non stéroïdiens et lésions digestives hautes : soigner en blessant ? J Afric HépatolGastrol 2016 ; 10(3) : 138-143.

10. Camara S. La restauration de la mastication à l'aide de la prothèse amovible au CHU-CNOS de Bamako. Thèsemed, Bamako, 2008; 77. 100p.

11. Association SOS Hépatite Mali. Communiqué de presse Bamako le 16 mars 2011 " www.soshepatited.org " (mise à jour le 4 Aout 2015).

12. Traoré A M. Extractions dentaires : étiologies et dents concernées au service d'odontologie chirurgicale du CHU-CNOS de Bamako. Thèsemed, Bamako, 2008 ; 486. 113p.

13. Diouf M, Boëtsch G, Tal-Dia A, Patrick T, JeanJacques $B$. Pathologie digestive et état buccodentaire chez les populations rurales de Ferlo au Sénégal. J odont stomotol tropicale 2013 ; 36(141) : 43-48.

14. Mbodj EB, DioufM, Faye D, Ndiaye A, Seck MT, Ndiaye Cet al. Réhabilitation prothétique : évaluation des besoins en prothèse dans les cabinets dentaire du Sénégal. Bull SOC PatholExo 2011; 104 :355-356. 
Liste des tableaux :

Tableau I : répartition des patients selon le sexe

\begin{tabular}{lcc}
\hline Sexe & Effectif & $\%$ \\
\hline Féminin & 42 & 42,0 \\
Masculin & 58 & 58,0 \\
Total & 100 & 100,0 \\
\hline
\end{tabular}

Tableau II : répartition des patients selon les tranches d'âge

\begin{tabular}{lcc}
\hline $\begin{array}{l}\text { Tranche d'âge } \\
\text { (ans) }\end{array}$ & Effectif & $\%$ \\
\hline $15-24$ & 8 & 8,0 \\
$25-34$ & 20 & 20,0 \\
$35-44$ & 24 & 24,0 \\
$45-54$ & 25 & 25,0 \\
\hline
\end{tabular}

\begin{tabular}{ccc}
\hline $55-64$ & 13 & 13,0 \\
$65-74$ & 4 & 4,0 \\
$75-84$ & 4 & 4,0 \\
$85-95$ & 2 & 2,0 \\
Total & 100 & 100,0 \\
\hline
\end{tabular}

Tableau IV : distribution des porteurs selon les pathologies

\begin{tabular}{lcc}
\hline Pathologies & Effectif & $\%$ \\
\hline Gastriques & 6 & 54,5 \\
Hépatique & 2 & 18,2 \\
OEsophagienne & 2 & 18,2 \\
VIH et opportuniste & 1 & 9,1 \\
Total & 11 & $\mathbf{1 0 0 , 0}$ \\
\hline
\end{tabular}

Tableau III : distribution de la population édentée selon les pathologies

\begin{tabular}{|l|c|c|c|c|}
\hline \multirow{2}{*}{ Pathologies } & \multicolumn{4}{|c|}{ Dents absentes en \% } \\
\cline { 2 - 5 } & 1 à 3 & 4 à 9 & 10 à 14 & $\geq 15$ \\
\hline Colorectale & 4,3 & 7,1 & 0,0 & 0,0 \\
\hline Gastrique & 43,5 & 10,7 & 16,7 & 42,9 \\
\hline Hépatique & 30,4 & 39,3 & 33,3 & 42,9 \\
\hline OEsophagienne & 4,3 & 3,6 & 16,7 & 0,0 \\
\hline VIH et opportunistes & 8,7 & 21,4 & 33,3 & 0,0 \\
\hline Autres * & 8,7 & 17,9 & 0,0 & 14,3 \\
\hline Total & 100,0 & 100,0 & 100,0 & 100,0 \\
\hline
\end{tabular}

Autres * : tumeur pancréatique : 3,6\%(4 à 9 dents), dystrophie cérébrale : 3,6\% (4 à 9 dent), paludisme grave : 8,7\% (1 à 3 dents); $3,6 \%$ (4 à 9 dents); plus de 15 dents (14,3\%), pneumopathie : $7,1 \%$ (4 à 9 dent). 